\title{
A correção da mordida cruzada posterior unilateral com desvio funcional melhora a assimetria facial?
}

\author{
Marco Antonio de Oliveira Almeida*, Catia Cardoso Abdo Quintão**, Ione Helena Vieira Portella Brunharo***,

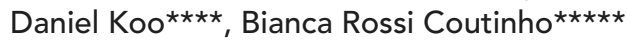

\section{Resumo}

Objetivo: avaliar, através de fotografias clínicas de 30 pacientes, as alterações ocorridas na assimetria labial presente em pacientes portadores de mordida cruzada posterior unilateral funcional (MCPUF), após a correção ortodôntica. Métodos: para quantificar essas modificações, foram mensuradas as áreas dos quadrantes labiais antes (T1) e após o tratamento (T2). Os lábios superiores e inferiores foram subdivididos em 4 quadrantes e as suas áreas foram medidas e expressas como percentagem da sua área total. A quantidade de correção da assimetria foi obtida calculando a diferença no percentual da área entre os quadrantes do lábio em T1 e T2. Resultados: os resultados demonstraram haver uma melhora significativa na assimetria observada inicialmente nestes pacientes do lado onde o desvio postural da mandíbula se apresentava.

Palavras-chave: Mordida cruzada funcional. Dentadura mista. Assimetria facial.

\section{INTRODUÇÃO}

A mordida cruzada posterior é considerada como a relação dentária anormal no sentido vestibulolingual quando ocorre a oclusão dos $\operatorname{arcos}^{8}$, podendo ser classificada como esquelética, dentária ou funcional. A redução na largura do arco dentário superior é a causa mais frequente ${ }^{1,14,19}$ e, dependendo do grau de contração, a mordida cruzada será uni ou bilateral. A mordida cruzada posterior unilateral (MCPU) é a que ocorre com mais frequência, sendo resultante de um deslocamento lateral da mandíbula para uma posição anormal, porém mais confortável para o paciente ${ }^{2,7,19}$. A mordida cruzada posterior unilateral funcional (MCPUF) se baseia na presença de um desvio mandibular assimétrico da posição de relação cêntrica para a de máxima intercuspidação e é normalmente estabelecida com a irrupção dos dentes decíduos $^{7,12}$. A MCPU tem uma prevalência relativamente alta e varia entre 8 e 16\% das crianças na dentadura mista ${ }^{20}$.

Existe um consenso que a MCUPF deva ser corrigida assim que for diagnosticada ${ }^{1,8,16}$, uma vez que a correção espontânea é improvável e possibilita a irrupção anormal dos dentes permanentes e interfere no crescimento dos arcos dentários ${ }^{8,10,19}$. Quando essa má oclusão não é corrigida durante as fases de crescimento, pode ocorrer uma assimetria estrutural, cujo tratamento se torna bem mais complexo ${ }^{3,8}$. Pacientes com MCPUF possuem assimetria

\footnotetext{
* Pós-doutorado UNC - Chapel Hill.

** Doutor em Ortodontia pela UFRJ.

*** Doutor em Ortodontia pela FO-UERJ.

$\star \star \star \star *$ Especialista em Ortodontia pela FO-UERJ.

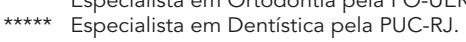


mandibular meramente posicional e, devido a esse fato, a expansão maxilar é considerada o tratamento de escolha para que a mandíbula retorne à posição normal ${ }^{18}$. Dentre os aparelhos mais utilizados na correção precoce da MCP está o arco em "W" ou aparelho de Porter ${ }^{6}$. Esse aparelho oferece certas vantagens, como a flexibilidade em seu ajuste, fácil higienização pelo paciente e ação independente da colaboração do paciente, por se tratar de um aparelho fixo ${ }^{7}$.

A assimetria apresentada no plano transverso dos arcos dentários é, segundo Proffit, Phillips e Dann $^{15}$, a mais perceptivel aos olhos do paciente e do observador. A assimetria na face e na dentição é um fenômeno que ocorre naturalmente e, na maioria dos casos, só pode ser observada pela comparação das partes homólogas da face. A arquitetura dos tecidos faciais é o fator mais importante que enfatiza a presença de uma assimetria com componente esquelético ou não.

O terço inferior da face, em geral, e a arquitetura labial, em particular nos pacientes com MCPUF, aparecem quase simétricos quando os pacientes são manipulados para determinar os contatos posteriores, ou quando os lábios estão distanciados, no repouso. Entretanto, o desvio mandibular e a assimetria labial podem ser notados a cada fechamento mandibular, deglutição ou, ocasionalmente, na fala ${ }^{11}$.

Gazit-Rappaport, Weinreb e Gazit ${ }^{5}$ quantificaram o grau de assimetria labial presente em pacientes portadores de MCPUF pré e pós-tratamento, a partir de fotografias da face. Para tal, os autores dividiram os lábios em 4 quadrantes e mediram a área de cada um, sendo esta expressa em percentagem da superfície total dos lábios. Os pacientes do grupo de estudo foram comparados a um grupo controle e apresentaram uma melhora da assimetria labial quantitativa e visual. Após a correção da MCPUF, os valores da superfície labial de cada lado aproximaram-se de $50 \%$. Seus resultados traduziram uma forte correlação da presença da má oclusão com a assimetria labial.
A arquitetura dos tecidos moles faciais é o fator mais importante que destaca a presença de uma assimetria com ou sem componente esquelético ${ }^{5}$. Observando-se a literatura, em relação às assimetrias transversas, há um maior enfoque das estruturas esqueléticas dos maxilares ${ }^{4,17}$, porém, poucos estudos clínicos foram publicados analisando a assimetria labial nesses pacientes.

\section{MATERIAL E MÉTODOS}

Foram selecionados, por 3 ortodontistas na cidade do Rio de Janeiro, 30 pacientes (13 meninos e 19 meninas) com idade média de 9,5 \pm 1,2 anos, portadores de MCFU envolvendo pelo menos dois dentes posteriores no quadrante $\left(1^{\circ}\right.$ molar permanente mais um elemento decíduo do hemiarco, pelo menos). O diagnóstico da assimetria mandibular postural foi realizado clinicamente quando os arcos encontravam-se em máxima intercuspidação e confirmado pelas fichas clínicas e modelos de estudo. Dessa forma, para ser incluído na amostra, era necessário que, em posição de repouso, o desvio da linha média não fosse visível e que, ao ocluir, o paciente apresentasse uma interferência que causasse um desvio lateral da mandíbula ${ }^{18}$.

Os pacientes deveriam apresentar fotografias de frente antes e após o tratamento, e com lábios selados passivamente, com os dentes em máxima intercuspidação habitual (MIH) (Fig. 1). Todos apresentavam um desvio do lábio inferior para o lado cruzado, resultando num aspecto de assimetria labial. Esses pacientes foram tratados com o aparelho de Porter ou com o aparelho disjuntor tipo Hyrax, ainda na dentadura mista.

As fotografias foram escaneadas com alta definição no scanner Epson Expression 1680 (Epson American Inc., Long Beach, Califórnia, EUA). Cada paciente gerou uma imagem digitalizada frontal ao início (T1) e ao final do tratamento (T2). Para padronização dessas imagens, optou-se pela manutenção da distância bipupilar em T1 e T2. No programa Autocad (Autodesk Inc., San Rafael, EUA, 2005), mediu-se essa distância para cada 

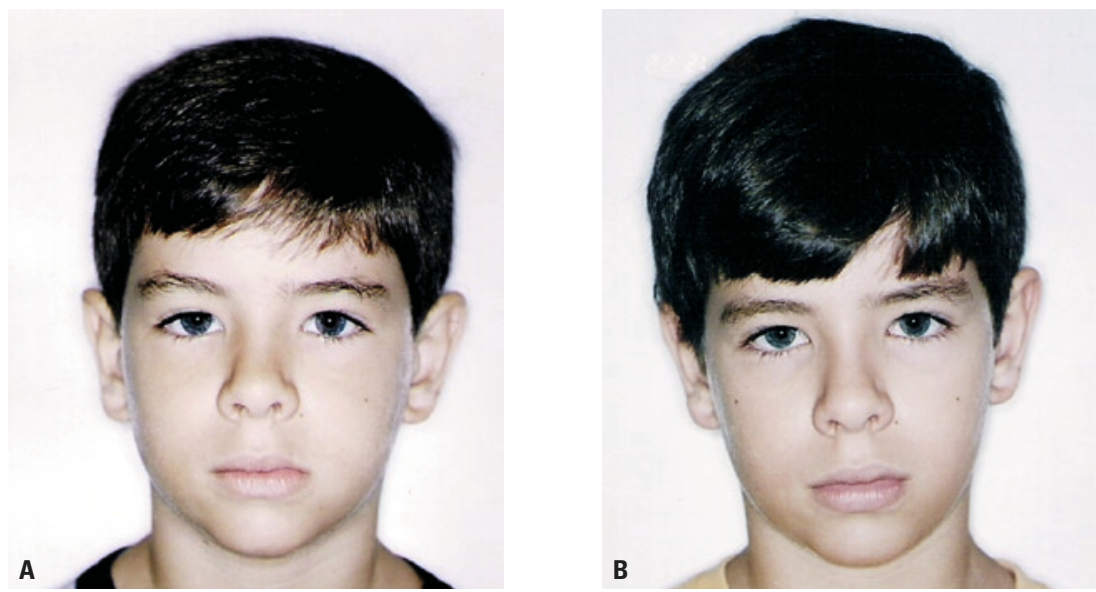

FIGURA 1 - A) Vista frontal de um paciente do estudo demonstrando o grau de assimetria labial pré-tratamento. B) Vista frontal demonstrando a melhora da assimetria visual dos lábios obtida pós-tratamento.

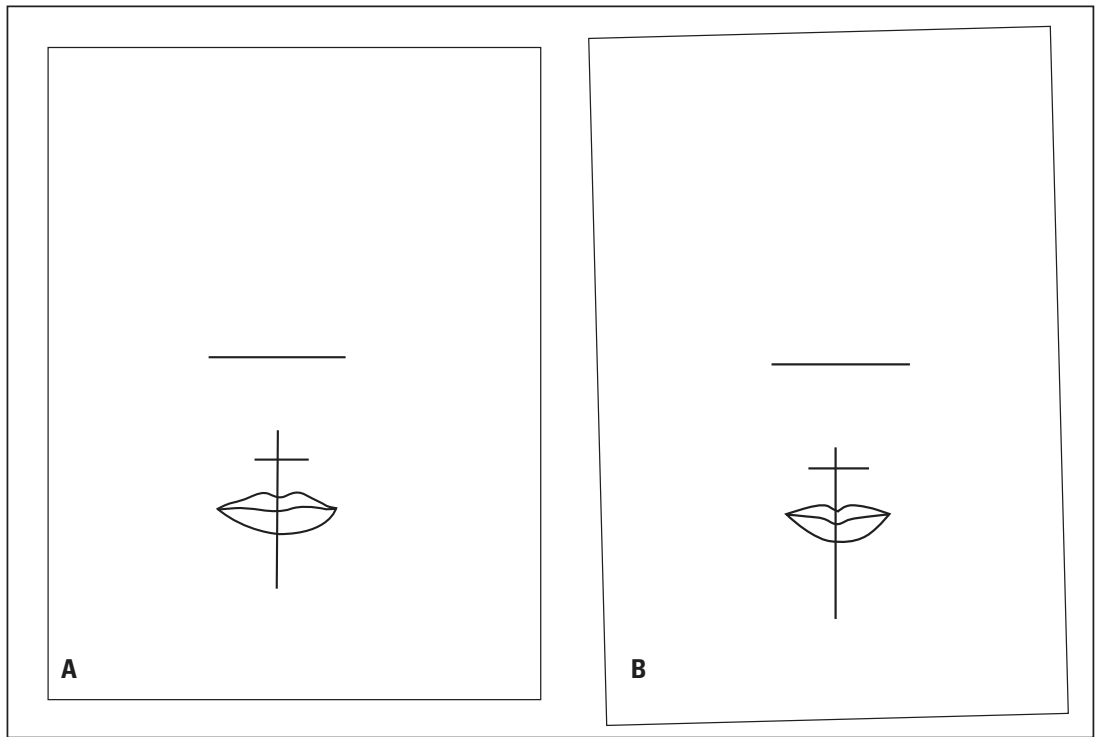

FIGURA 2 - Exemplo da imagem obtida no programa Autocad para a leitura das áreas dos quadrantes labiais gerados após a correção da distância bipupilar de T1 e T2 e da inclinação da cabeça. Em B), observa-se o giro anti-horário do retângulo maior.

paciente, em T1 e em T2, onde essa imagem deveria ser corrigida por redução ou ampliação do seu tamanho. Da mesma maneira, uma possível correção da inclinação da cabeça foi realizada quando necessário, utilizando a ferramenta do programa Autocad que permitia colocar o plano transverso (Tragus, canto do olho, base nasal) paralelo ao chão (Fig. 2).

No mesmo programa de imagens, o terço inferior da face foi obtido pelo recorte da imagem e, para que os lábios fossem divididos em quadrantes, foi construída uma linha tangente à base do nariz e outra partindo do filtro labial em direção ao queixo (Fig. 3).

O contorno labial e a linha transversa que une os lábios foram cuidadosamente desenhados para criar, juntamente com a linha vertical, 4 quadrantes (Fig. 2), 2 formados pelo lábio superior e 2 pelo lábio inferior. A superfície da área de cada 

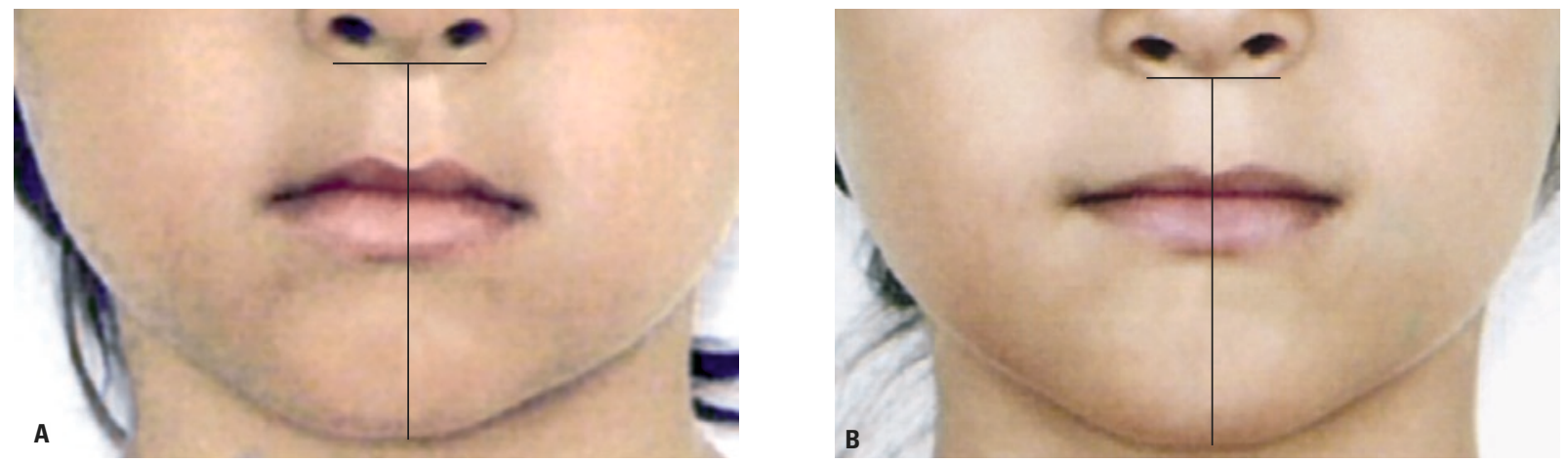

FIGURA 3 - Exemplo do terço inferior da face de uma paciente da amostra obtido a partir das fotografias dos pacientes em T1 e T2, onde foi construída uma linha tangente à base do nariz e outra partindo do filtro labial em direção ao queixo, a fim de dividir os lábios em 4 quadrantes labiais.

quadrante foi medida com o programa Autocad e os dados de cada quadrante foram expressos em percentagem da área total dos lábios superior e inferior.

Esse procedimento foi realizado por um mesmo operador. Para verificar a capacidade de reprodução do método, foi realizada a leitura dos quadrantes labiais de 8 pacientes, selecionados aleatoriamente e sem identificação, repetindo-se o procedimento 3 vezes com intervalo de 24 horas.

\section{RESULTADOS}

Verificou-se a concordância de Kappa igual a $8,7(\mathrm{k}=8,7)$ para o erro de método intraoperador, determinando estar apto para a repetição do método.

Os pacientes com MCPUF apresentaram uma considerável diferença na área dos quadrantes labiais no lado cruzado após o tratamento. O quadrante superior desse lado exibiu um aumento médio percentual da área $(\mathrm{T} 2=18,7 \%)$ comparandose com o valor inicial ( $\mathrm{Tl}=17,4 \%)$, na tabela 1 . No quadrante inferior do mesmo lado, ocorreu uma diminuição da área ao final do tratamento. Ao início, essa região tinha uma média de $34,7 \%$ e ao final de $32,5 \%$. Essa redução mostrou-se significativa em nível de 1\% (Tab. 1).

Esses resultados são corroborados pelas observações clínicas e pelo trabalho de Gazit-Rappaport, Weinreb e Gazit ${ }^{5}$, onde as mudanças na área do lábio inferior do grupo de estudo foram maiores que do lábio superior (Fig. 1, 2).

Quanto ao lado oposto ao desvio mandibular, onde a mordida cruzada não incidia, o lado superior, ao final do tratamento, apresentou um aumento médio percentual da área, passando de 0,168

TABELA 1 - Valores percentuais médios obtidos das áreas dos quadrantes labiais pré e pós-tratamento (teste $t$ - Student).

\begin{tabular}{|c|c|c|c|c|c|}
\hline quadrante & média & desvio-padrão & valor percentual & diferença média & $\mathbf{p}$ \\
\hline cruzado superior inicial (T1) & 0,174 & 0,0243 & 17,4 & \multirow{2}{*}{0,0128} & \multirow{2}{*}{$0,037^{* *}$} \\
\hline cruzado superior final (T2) & 0,187 & 0,0313 & 18,7 & & \\
\hline cruzado inferior inicial (T1) & 0,347 & 0,0269 & 34,7 & \multirow{2}{*}{$-0,0229$} & \multirow{2}{*}{$<0,001^{*}$} \\
\hline cruzado inferior final (T2) & 0,325 & 0,0403 & 32,5 & & \\
\hline normal superior inicial (T1) & 0,168 & 0,0236 & 16,8 & \multirow{2}{*}{0,0115} & \multirow{2}{*}{$0,012^{* *}$} \\
\hline normal superior final (T2) & 0,180 & 0,0349 & 18,0 & & \\
\hline normal inferior inicial (T1) & 0,309 & 0,0264 & 30,9 & \multirow{2}{*}{$-0,0014$} & \multirow{2}{*}{0,841} \\
\hline normal inferior final (T2) & 0,308 & 0,0411 & 30,8 & & \\
\hline
\end{tabular}

* Estatisticamente significativo ao nível de $1 \%,{ }^{* *}$ estatisticamente significativo ao nível de $5 \%$. 
$(16,8 \%)$ em Tl para 0,180 (18\%) em T2. Essa alteração foi estatisticamente significativa ( $\mathrm{p}$-valor $=$ 0,012). Já o quadrante inferior do lado não-cruzado, ao final do tratamento, não apresentou alteração estatisticamente significativa da área (Tab. 1).

Esses dados não concordam com o trabalho de Gazit-Rappaport, Weinreb e Gazit ${ }^{5}$, que mostrou - ao término da correção da MCPUF no lado cruzado - uma melhora no sentido da correção da assimetria labial, porém com diminuição do percentual médio da área do quadrante superior. Esses autores verificaram que o quadrante inferior deste lado apresentou um aumento do percentual médio da área.

O quadrante inferior desse lado, no trabalho em questão, apresentou um aumento e o superior do mesmo lado apresentou uma diminuição da área média percentual.

\section{DISCUSSÃO}

O ortodontista objetiva, ao término do tratamento ortodôntico, obter o equilíbrio e a harmonia facial. Geralmente as assimetrias dentoesqueléticas - e, em particular, as funcionais - resultam, quando não corrigidas, em alterações das estruturas musculares e adaptações articulares ${ }^{2,13}$. O consenso geral é que a mordida cruzada funcional pode ser corrigida assim que possível, para eliminar seu potencial para as mudanças adaptativas musculoesqueléticas ou, se já presente, para reduzi-las no futuro ${ }^{11}$.

Uma vez que os tecidos faciais se acomodam na dependência da configuração esquelética que os ossos faciais se apresentam, os lábios sofrem, também, essa influência. Diferentes formas labiais podem ser encontradas, variando em graus de espessura, seja por uma característica hereditária ou por um comprometimento dento-esquelético-funcional.

$\mathrm{Na}$ presença de uma MCPUF envolvendo até os caninos, por exemplo, os lábios têm nessa região um apoio dentário que promove uma assimetria das suas partes homólogas labiais. Nesse caso, o lábio inferior do lado cruzado tem um suporte dentário mais pronunciado, pelo elemento ou elementos protruídos, e mostra aumento da espessura, ao contrário do lábio superior, pela falta desse suporte. $\mathrm{Na}$ presença de um deslocamento funcional, portanto, é claro admitir que a assimetria mandibular desloca e empurra o lábio inferior para o mesmo lado. Observa-se, geralmente, alteração da simetria da comissura labial (Fig. 2).

A assimetria labial deverá sempre ser examinada quando o deslocamento total da mandíbula ocorrer previamente ao tratamento. Nos casos da MCPUF, numa posição de repouso, sem nenhum contato interdentário, a mandíbula situa-se mais centralizada e a assimetria não é tão evidente. $\mathrm{Lu}^{9}$ argumentou que somente assimetrias faciais maiores do que 3\% são clinicamente discerníveis, o que está de acordo com o trabalho de Gazit-Rappaport, Weinreb e Gazit ${ }^{5}$. Em seu estudo, a assimetria de até 3\% entre o lado direito e esquerdo não se fez clinicamente aparente no momento do exame.

No presente estudo, pode-se verificar na tabela 1 que o percentual médio da área para os lábios superiores foi cerca de $48,5 \%$ menor do que para os lábios inferiores.

Os lábios superiores, por serem mais finos do que os inferiores, proporcionalmente tiveram, após a correção da MCPUF, seus quadrantes direito e esquerdo se equivalendo. Observa-se que o lado cruzado, ao término do tratamento, registrou uma alteração de 0,187 e o lado normal de 0,180, ou seja, os valores das áreas tenderam a se equivaler.

A assimetria labial pode ser usada como uma ferramenta de auxílio no diagnóstico ortodôntico. O profissional deve estar apto a reconhecer quando a assimetria labial ocorre e, assim, procurar objetivar a sua correção no tratamento. A análise quantitativa da área do lábio nas assimetrias resultou em uma valiosa ajuda para avaliação dos efeitos resultantes do tratamento.

Estudos futuros são necessários para mostrar se as alterações ocorridas após o tratamento precoce dessa má oclusão permitem uma melhora da face, tornando-se uma ferramenta útil para a mensuração da assimetria labial. 


\section{CONCLUSÕES}

A correção da MCPUF estabeleceu a simetria labial, tanto no aspecto visual quanto no quantitativo.

O foco na assimetria labial pode incentivar pacientes ainda na dentadura mista a buscar o tratamento precoce, prevenindo o envolvimento esquelético, muscular e complicações dentárias da má oclusão na fase adulta.

\title{
Does the correction of a functional posterior unilateral crossbite improve the facial asymmetry?
}

\begin{abstract}
Aim: To evaluate, analyzing clinical photographs of 30 patients, the changes occurred, after orthodontic correction, on the labial asymmetry in patients with unilateral posterior functional crossbite. Methods: To quantify this changes, the area of the labial quadrants and their surface area were measured before (T1) and after (T2) the treatment. Upper and lower lips were subdivided in 4 quadrants, and their surface area were measured and expressed in percentage of the total surface area. The degree of correction was obtained by calculating the difference in the percentile of the surface area among the quadrants in T1 and T2. Results: The results showed a significant improvement of the asymmetry on the mandibular shift side.
\end{abstract}

Keywords: Functional crossbite. Mixed dentition. Facial asymmetry.

\section{REFERÊNCIAS}

1. BELL, R. A.; LECOMPTE, E. J. The effects of maxillary expansion using a quad-helix appliance during the deciduous and mixed dentitions. Am. J. Orthod., St. Louis, v. 79, no. 2, p. 152-160, 1981.

2. BISHARA, S. E.; BURKEY, P. S.; KHAROUF, J. G. Dental and facial asymmetries: a review. Angle Orthod., Appleton, v. 64, no. 2, p. 89-98, 1994.

3. CAPELOZZA FILHO, L.; SILVA FILHO, O. G. Expansão rápida da maxila: considerações gerais e aplicação clínica. In: INTERLANDI, S. (Org.). Ortodontia: bases para iniciação. 3. ed. São Paulo: Artes Médicas, 1994. p. 393-418.

4. FORSBERG, C. T.; BURSTONE, C. J.; HANLEY, K. J. Diagnosis and treatment planning of skeletal asymmetry with the submento-vertical radiograph. Am. J. Orthod., St. Louis, v. 85, p. 224-237, 1984.

5. GAZIT-RAPPAPORT, T.; WEINREB, M.; GAZIT, E. Quantitative evaluation of lip symmetry in functional asymmetry. Eur. J. Orthod., Oxford, v. 25, no. 5, p. 443-450, 2003.

6. HAYASAKI, S. M.; CANTO, G. D. L.; HENRIQUES, J. F. C. et al. A importância da correção precoce da mordida cruzada posterior. Rev. Dental Press Ortodon. Ortop. Facial, Maringá, v. 3, n. 6, p. 30-34, 1998.

7. KING, D. L. Functional posterior crossbite in the deciduous and early mixed dentition. Gen. Dent., Chicago, v. 26, no. 1 , p. 36-40, 1978.

8. KUTIN, G.; HAWES, R. R. Posterior crossbites in the deciduous and mixed dentitions. Am. J. Orthod., St. Louis, v. 56, no. 5 , p. 491-504, 1969.

9. LU, K. H. Harmonic analysis of the human face. Biometrics, Washington, D. C., v. 21, p. 491-505, 1965.

10. McDONALD, R. E.; AVERY, D. R. Odontopediatria. 4. ed. Rio de Janeiro: Guanabara Koogan, 1986.

11. MONGINI, F.; SCHMID, W. Treatment of mandibular asymmetry during growth: a longitudinal study. Eur. J. Orthod., Oxford, v. 9, p. 51-67, 1987.
12. MOYERS, R. E. Ortodontia. 4. ed. Rio de Janeiro: Guanabara Koogan, 1991.

13. PIRTTINIEMI, P. Normal and increased functional asymmetry in the craniofacial area. Acta Odontol. Scand., Oslo, v. 56, no. 6, p. $342-345$, Dec. 1998

14. PROFFIT, W. R.; FIELDS, H. W. Ortodontia contemporânea. 2. ed. Rio de Janeiro: Guanabara Koogan, 1995.

15. PROFFIT, W. R.; PHILLIPS, C.; DANN, C. Who seeks surgicalorthodontic treatment? Int. J. Adult Orthodon. Orthognath. Surg., Chicago, v. 5, p. 153-160, 1990.

16. PURCELL, P. D. Effectiveness of posterior crossbite correction during the mixed dentition. J. Pedod., Boston, v. 9, no. 4, p. 302-311, 1985.

17. ROSE, J. M.; SADOWSKY, C.; BEGOLE, E. A. et al. Mandibular skeletal and dental asymmetry in Class II subdivision malocclusions. Am. J. Orthod. Dentofacial Orthop., St. Louis, v. 105, p. $489-495,1994$

18. SANTOS PINTO, A.; MONNERAT, M. E. Avaliação da relação côndilo-fossa articular em crianças com mordida cruzada posterior unilateral funcional na fase da dentadura mista. Rev. SOB, Rio de Janeiro, v. 2, n. 2, p. 30-36, 1992.

19. SCHRÖDER, U.; SCHRÖDER, I. Early treatment of unilateral posterior crossbite in children with bilaterally contracted maxillae. Eur. J. Orthod., Oxford, v. 6, no. 1, p. 65-69, 1984.

20. THILANDER, B.; WAHLUND, S.; LENNARTSSON, B. The effect of early interceptive treatment in children with posterior crossbite. Eur. J. Orthod., Oxford, v. 6, p. 25-34, 1984.

\section{Endereço para correspondência}

Bianca Rossi

Rua Joana Angélica, 35/301, Ipanema

CEP: 22.420-030 - Rio de Janeiro/RJ

Email: bia.rossi@uol.com.br 